\title{
Heat Transfer from a Cylinder in the Production and in the Decay Regions of Grid-Generated Turbulence
}

\author{
Gianfrancesco Melina $^{1 *}$, Paul J. K. Bruce ${ }^{1}$, Geoffrey F. Hewitt ${ }^{2}$, J. Christos Vassilicos ${ }^{1}$ \\ ${ }^{1}$ Department of Aeronautics \\ ${ }^{2}$ Department of Chemical Engineering \\ ${ }^{1,2}$ Imperial College London, South Kensington Campus, SW7 2AZ London, UK \\ *g.melina13@imperial.ac.uk
}

\section{Extended Abstract}

The effects of grid-generated turbulence on the local heat transfer coefficient around the circumference of a cylinder in crossflow are investigated experimentally in a wind tunnel. A thin Inconel foil $(25 \mu \mathrm{m}$ thick $)$ is wrapped around the central portion of the cylinder and is resistively heated with a direct electrical current, a technique approximating a uniform heat flux boundary condition on the cylinder's wall [1]. The wall temperature $T_{w}$ is measured around the cylinder with thermocouples installed underneath the heater foil. Three grids with different geometry and different blockage ratios $\sigma_{g}$ are placed at the inlet of the wind tunnel: a regular square-mesh grid (RG60) with $\sigma_{g}=32 \%$, a fractal square grid (FSG17) with $\sigma_{g}=25 \%$, and a single square grid (SSG) with $\sigma_{g}=20 \%$. The grid-generated turbulent flows are documented first without cylinder in terms of turbulence intensity $T u$ and integral length scale $L_{u}$ using hot-wire anemometry along the centreline. The cylinder is subsequently placed at several streamwise distances $x$ from each grid and the heat transfer measurements are performed around the centreline circumference. The Reynolds number $R e$, based on the cylinder's diameter $D$, varies approximately between 10800 and 48800 .

For the same turbulence parameter $T u R e^{0.5}$ [2], the Frossling number $N u_{0} / R e^{0.5}\left(N u_{0}\right.$ is the Nusselt number at the cylinder's front stagnation point) is highest for RG60, as a result of the lowest ratios $L_{u} / D$ produced by this grid, in agreement with [3]. The maximum enhancement of the circumferentially averaged Nusselt number $N u_{a v g}$, with respect to its value under laminar free-stream conditions $N u_{\text {lam }}$, occurs in the proximity of $x_{\text {peak }}$, where $T u$ is maximum, and it increases with $R e$. The maximum value of $N u_{a v g} / N u_{l a m}$ is higher for SSG than for FSG17, despite SSG having a lower $\sigma_{g}$. This is explained by the larger values of $T u$ in the production region of SSG, which are achieved by increasing the ratio $t_{0} / L_{0}$ [4] with respect to FSG17, where $t_{0}$ and $L_{0}$ are the thickness and the length of the largest bars of the grid respectively. However the rate of decrease of $N u_{\text {avg }} / \mathrm{Nu} u_{\text {lam }}$ with $x$ is lower for FSG17, thus reflecting the slower decay of $T u$ along $x$ for this grid. At large distances from the grids, the values of $N u_{\text {avg }}$ are appreciably higher for SSG and for FSG17 than for RG60, despite the latter having a higher $\sigma_{g}$.

The SSG, and to a greater extent the FSG17, exhibit a prolonged turbulence production region, where the turbulence intensity $T u$ increases with $x$ before decaying. This allows the unprecedented opportunity of comparing the angular heat transfer profiles in the production and in the decay regions for same values of $T u$. It is found that on the forward part of the cylinder, where a laminar boundary layer exists, the ratio $\mathrm{Nu} / \mathrm{R} e^{0.5}$ is lower in the production region of the grids, especially for SSG. This can be related to (i) a higher energy of the vortex shedding from the bars of the grids, especially for SSG for which the shedding is stronger, and to (ii) a higher intermittency of the flow in the production region which mitigates the effects of $T u$ on the heat transfer enhancement [5]. On the downstream side of the cylinder, where the flow is separated, the heat transfer recovery parameter $\mathrm{Nu} / \mathrm{N} u_{0}$ is significantly higher in the production region. This could suggest a decrease of the vortex formation length downstream of the cylinder, due to interaction between the cylinder's wake and the wakes originating from the largest bars of the grid, which in the production region have not merged yet [6].

\section{References}

[1] S. Sanitjai and R. J. Goldstein, "Forced convection heat transfer from a circular cylinder in crossflow to air and liquids," Int. J. Heat Mass Transfer, vol. 47, pp. 4795-4805, 2004. 
[2] G. W. Lowery and R. I. Vachon, "The effect of turbulence on heat transfer from heated cylinders," Int. J. Heat Mass Transfer, vol. 18, pp. 1229-1242, 1975.

[3] P. Gandavarapu and F. E. Ames, "The influence of leading edge diameter on stagnation region heat transfer augmentation including effects of turbulence level, scale and Reynolds number," J. Turbomach., vol. 135:011008, 2013.

[4] R. Gomes-Fernandes, B. Ganapathisubramani and J. C. Vassilicos, "Particle image velocimetry study of fractalgenerated turbulence," J. Fluid Mech., vol. 711, pp. 306-336, 2012.

[5] N. T. Van Dresar and R. E. Mayle, "A quasi-steady approach of wake effects on leading edge transfer rates," J. Turbomach., vol. 111, pp. 483-490, 1989.

[6] N. Mazellier and J. C. Vassilicos, "Turbulence without Richardson-Kolmogorov cascade," Phys. Fluids, vol. 22:035103, 2010 